\title{
Low carbohydrate diet and improved glycaemic control in a patient with type one diabetes
}

\author{
Mirjam Eiswirth', Ewan Clark² and Michael Diamond² \\ 1University of Edinburgh, Edinburgh, UK and 2University of Edinburgh Health Centre, Edinburgh, UK \\ Correspondence \\ should be addressed \\ to M Diamond \\ Email \\ diamond.michael@gmail.com
}

\section{Summary}

We present the case of an adult female with type 1 diabetes, whose $\mathrm{HbA} 1 \mathrm{c}$ was trending at $58 \mathrm{mmol} / \mathrm{mol}(7.5 \%) \mathrm{for}$ the past 3 years. In August 2016, she reduced her total daily carbohydrate intake to $30-50 \mathrm{~g}$ and adjusted her other macronutrients to compensate for the calorific deficit. Her HbA1c fell to $34 \mathrm{mmol} / \mathrm{mol}$ (5.3\%) by January 2017 and average daily blood glucose readings decreased significantly from 10.4 to $6.1 \mathrm{mmol} / \mathrm{L}$. Moreover, she observed a marked reduction of average daily glucose variability. Notably, there were no significant episodes of hypo- or hyperglycaemia and her lipid profile remained static. Subjectively, she described an improvement in her quality of life and the dietary transition was extremely well tolerated. We discuss these findings in detail and the potential clinical benefits for patients with type 1 diabetes that can be gained by following a low carbohydrate diet.

\section{Learning points:}

- A low carbohydrate diet was found to substantially reduce HbA1c values and blood glucose (BG) variability, as well as causing a significant reduction in average daily glucose values in a patient with T1DM.

- Although further research is warranted, low carbohydrate diets in patients with T1DM have the potential to positively impact long-term morbidity and mortality through reduction of BG variability and average daily BG values.

- The diet was well tolerated and not associated with any adverse effects within this study.

\section{Background}

Type 1 diabetes mellitus (T1DM) is an autoimmune disease, resulting in the self-destruction of pancreatic islet beta cells. The eventual outcome is cessation of endogenous insulin production in response to rising blood glucose (BG) levels. It is associated with a significant pathological burden, and has been well established that poor glycaemic control correlates with increasing morbidity and mortality (1).

Glycosylated haemoglobin (HbA1c) is a marker of glycaemic control, representing an approximate average of BG levels over a three-month period. The non-diabetic population has a HbA1c of less than $42 \mathrm{mmol} / \mathrm{mol}(6.0 \%)$. However, in T1DM, target levels at or below $48 \mathrm{mmol} / \mathrm{mol}$ (6.5\%) are generally considered desirable (2). Achieving good glycaemic control can be an arduous process for patients, as it requires careful and educated matching of carbohydrate intake with insulin. Physical activity, exogenous stress or hormonal change can significantly influence insulin requirement and BG homeostasis (3). Poor control is also often represented by erratic daily BG levels. This in turn is frequently associated with an elevated HbA1c, a hallmark of chronic hyperglycaemic 
insult. More acutely, steep BG variations greatly increase the potentially mortal risks of severe hypoglycaemia and/or ketoacidosis.

Before the advent of exogenous insulin, the only medical treatment available was a strict adherence to a low carbohydrate diet. Today, insulin is most commonly injected as a long-acting basal regime combined with short-acting boluses for corrective doses. The extracorporeal subcutaneous insulin pump and the recent advent of glucose monitoring sensors (both flash and continuous) have facilitated a closer approximation of normal physiology in type 1 patients. Despite these advances, we are still struggling to achieve normal BG control in this population. Moreover, there is limited evidence examining the impact of a very low carbohydrate diet on overall glycaemic control (HbA1c, average BG and variability) in patients with T1DM.

\section{Case presentation}

The patient presented here is a female in her twenties who was diagnosed with T1DM at the age of five years. She commenced insulin pump therapy at the age of 13 years and had stable control of her diabetes over the past decade. The patient had previously attended several courses similar to DAFNE (dose adjustment for normal eating). HbA1c readings from 2013 until mid-2016 ranged from 54 to $62 \mathrm{mmol} / \mathrm{mol}(7.1-7.8 \%)$, and she had no end-organ diabetic complications. Her insulin pump was a Medtronic $640 \mathrm{~g}$ and BGs were monitored using a Freestyle Libre metre. When BG readings were higher than $10 \mathrm{mmol} / \mathrm{L}$ or lower than $3.5 \mathrm{mmol} / \mathrm{L}$, she would routinely cross-check with a Bayer Contour Next monitor.

She presented in December 2016 for a routine appointment in primary care. Her HbA1c had substantially decreased and on review her daily BGs typically fell within a narrower range with less marked fluctuation between readings. She was also recording gentler graded changes in BGs and much lower postprandial extremes. The change in glycaemic control was attributed to an intentional reduction, but not exclusion, of her total daily carbohydrate intake. Of note, no significant hypoglycaemic events were recorded over the preceding 5 months. Subjectively, her quality of life was better, and the side effects pertaining to the extremes of poorly regulated glucose levels had practically abated. She observed no adverse physiological effects of this diet on her activities of daily living. A small loss of $3 \mathrm{~kg}$ was noted over the timeframe of the study, and her BMI at the time of presentation was 23.1 .

\section{Investigation}

\section{Data collection}

Carbohydrate and calorie content of every meal were documented from March 2016 to March 2017, as were insulin intake and activity levels. BG readings $(\mathrm{mmol} / \mathrm{L})$ were recorded using her Freestyle Libre and a Bayer Contour Next monitor. HbA1c values $(\mathrm{mmol} / \mathrm{mol})$ were obtained from routine clinical monitoring.

\section{Treatment}

\section{Dietary modification}

From the 1st of August 2016, the patient reduced her total daily consumption of carbohydrates to approximately $30-50 \mathrm{~g}$. To ensure adequate caloric and nutritional intake, she increased her other macronutrients (protein, fibre and fat) accordingly.

\section{Outcome and follow-up}

Collectively, the data presented in this study show a significant reduction in average daily BGs as well as a marked reduction in BG variability and $\mathrm{HbA1c}$ values over a 6-month period. These changes were facilitated by a reduction in carbohydrate intake to an amount of $30-50 \mathrm{~g}$ daily.

Figure 1 plots the average daily BG values, expressed as a percentage of daily caloric intake against time. There was a reduction of both inter-daily variability and average daily BG values. Prior to the dietary modification, the patient's BGs were trending at $10.4 \mathrm{mmol} / \mathrm{L}$ on a daily basis. However, after the intervention 6 months later, this had improved to a daily average of $6.1 \mathrm{mmol} / \mathrm{L}$ (Fig. 2). For comparative reference, fasting BG levels in nondiabetic individuals are less than $7 \mathrm{mmol} / \mathrm{L}$. There was also a markedly reduced daily BG variability between the control and December values (27\%). Figure 3 represents the patient's HbA1c values over a 3-year period, showing a sharp decline in the HbA1c well below the diagnostic cut-off for diabetes $(42 \mathrm{mmol} / \mathrm{mol}$ or $6.0 \%)$. Notably, the patient's total daily requirements for insulin were also reduced from 50 to 30 units. In summary, these data collectively demonstrate that not only was there a transition in the average BGs to a normal range, but the net variability was greatly reduced and HbA1c was normalised to a non-diabetic level.

It is important to note that when the described dietary change was effected, the patient did not experience any 


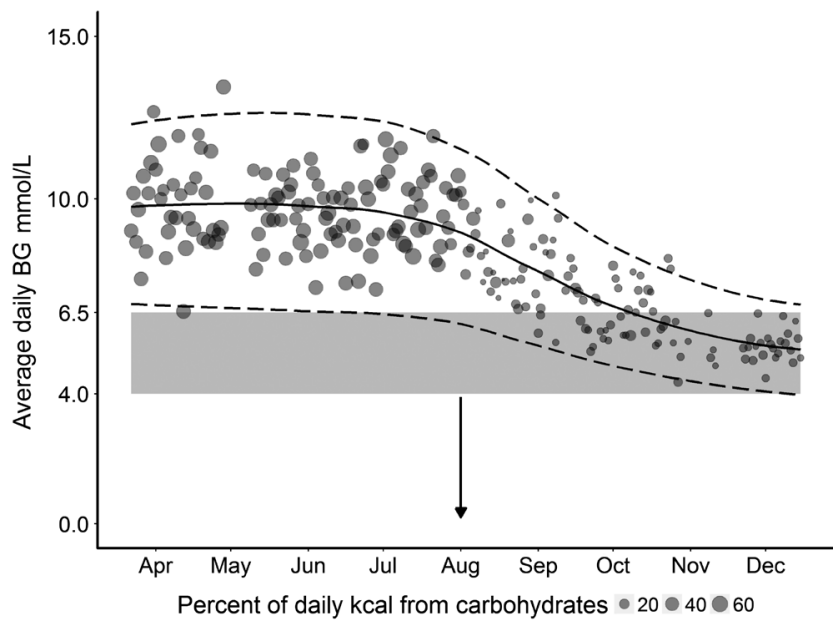

Figure 1

Average daily BG readings with standard deviation (s.D.) over 9 months, showing the percentage of carbohydrates of total daily kcal intake. The $x$ axis depicts the timeframe of the study and the $y$ axis the blood glucose readings in $\mathrm{mmol} / \mathrm{L}$. Average daily BG readings are plotted as circles, with a larger circle representing a proportionally larger percentage of carbohydrate intake (of total kcal) for that day. The mean and S.D. for the data are indicated by solid and dashed lines respectively. The lower greyed area shows an ideal target BG range of $4-6.5 \mathrm{mmol}$.

significant episodes of hypoglycaemia. Additionally, her hyperglycaemic events were infrequent, less marked and of shorter duration than those she recorded previously. Interestingly, it also emerged that the patient found she had to make insulin dose adjustments for high protein/fat meals, as they consistently caused a greater postprandial rise in BG than would be expected from the calculated carbohydrate load alone. No episodes of diabetic ketoacidosis were experienced by the patient. Overall, her random ketone measurements were reported in the range

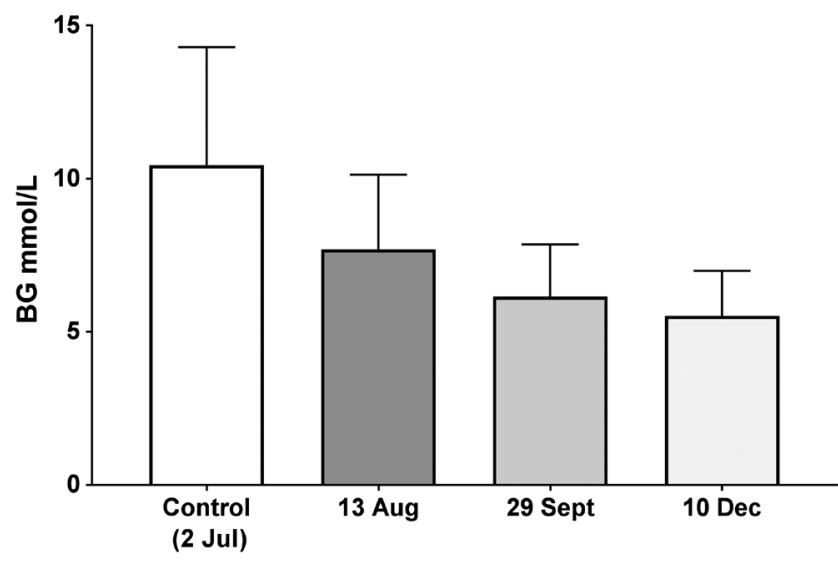

Figure 2

Average weekly BG readings across four study time points. Each column is an average of that week's total BG readings (mean \pm s.D.). The pre-diet group is labelled control.

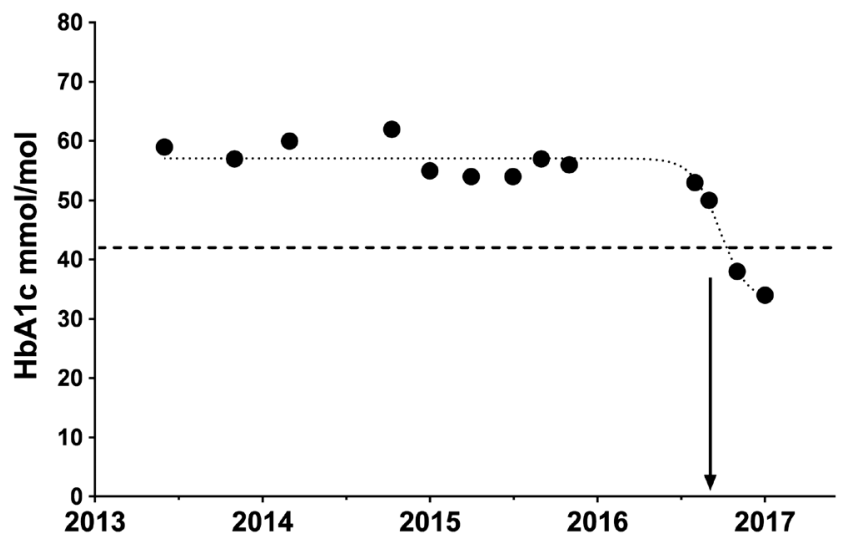

Figure 3

Graphical representation of $\mathrm{HbA} 1 \mathrm{c}$ readings over the past 3 years. The horizontal dashed line at $42 \mathrm{mmol} / \mathrm{mol}(6.0 \%)$ indicates the clinical upper limit in a non-diabetic individual. The vertical arrow notes the start of her dietary change on the 1st Aug 2016. $\mathrm{HbA} 1 \mathrm{c}$ was trending at $58 \mathrm{mmol} / \mathrm{mol}$ $(7.5 \%)$ prior to the dietary intervention. There was a reduction to 50 $(6.7 \%)$ in late September 2016, $38(5.6 \%)$ in November 2016 and $34 \mathrm{mmol} /$ $\mathrm{mol}(5.3 \%)$ in January 2017.

of $0.3-0.6 \mathrm{mmol} / \mathrm{L}$. The patient did report two episodes of a mild to moderate ketosis. On review, these were likely attributable to a miscalculation or a basal morning dose (ketones of $1.5 \mathrm{mmol} / \mathrm{L}$ ) and a prolonged exercise bout of three hours (ketones of $2.2 \mathrm{mmol} / \mathrm{L}$ ). Her other biochemical and haematological values remained static, with no adverse changes to her lipid profile (TC 4.0, LDL 2.1 ), and she described a marked improvement in her quality of life (see 'Patient perspective').

\section{Discussion}

The findings of this study clearly demonstrate that the impact of dietary manipulation on glycaemic control cannot be understated. The near normalisation of glycaemic control in this patient is likely multifactorial, and we propose several mechanisms underlying this. In general, a lower carbohydrate intake will inevitably lead to a proportionally smaller rise in BG. A smaller titration of insulin is consequently required, with a lower risk of over-correcting and in turn a reduction in overall glycaemic variability. Furthermore, with lower carbohydrate intake, muscular and liver stores of glycogen are less likely to be saturated. Thus, the maintenance of glucose homeostasis through active glycogenesis may contribute an important additional buffer to glycaemic control. Evidence from previous studies support this postulation, demonstrating that a glycogen-deplete state facilitates a more rapid uptake of circulating BG into muscular stores (4). 
We acknowledge the limitation of this case study, primarily as it is confined to the observational data from one patient. However, previous research corroborates our findings. Krebs et al. (5) examined the effects of a low carbohydrate diet in patients with T1DM over 12 weeks. Dietary intake was restricted to an average $100 \mathrm{~g}$ per day and subjects noted an average decrease in HbA1c from 63 to $55 \mathrm{mmol} / \mathrm{mol}$ (7.9-7.2\%). In 2006, Nielsen demonstrated that a daily intake of $70-90 \mathrm{~g}$ of carbohydrate significantly reduced hypoglycaemic events and lowered HbA1c from 58.5 to $46.4 \mathrm{mmol} / \mathrm{mol}$ (7.5-6.4\%) after 3 months (6). Although not looking at absolute reduction in carbohydrate intake, a Cochrane review in 2009 (7) scrutinised 11 randomised control trials looking at the impact of low glycaemic index diets in diabetic management. The collective findings were lower average HbA1c values and a reduced frequency of hypoglycaemic and hyperglycaemic events. Finally, our study also demonstrated a clear reduction in daily BG variability, a measure which has been proposed to play a key role in the pathology of end-organ damage in diabetes (8). Although the referenced evidence supports our findings, our study is the first to observe a collective normalisation of glycaemic control (average BM, variability and $\mathrm{HbA1c}$ ) with a very low carbohydrate diet.

We would caution that any such intervention be undertaken in a clinically supervised manner. Biochemically, a reduction in carbohydrate intake and subsequent downtitration of insulin, increases the use of ketone bodies as an energy source. In patients with type 1 diabetes, this can potentiate the risks for diabetic ketoacidosis. In this case, flash glucose monitoring facilitated a safer environment in which to observe regular changes in daily BGs. Furthermore, the pump allowed careful titration of insulin according to dietary intake as well as baseline circadian variation. A potential obstacle to applying our findings to clinical practice is that a strict low carbohydrate diet may have problems of adherence; a fact to which previous researchers have alluded $(6,7)$. However, with all lifestyle change it may be that gradual dietary modification, ideally supported by a dietician, could facilitate an easier and sustained transition.

An interesting observation of this study was the patient had to make additional adjustments in her insulin dose for very high protein/fat content meals as they consistently caused a greater postprandial rise in BG than would have been expected from the calculated carbohydrate load alone. A recent systematic review of 14 clinical studies examined the impact of fat and protein on postprandial BG readings and repeatedly found that meals high in protein/fat required higher boluses of insulin than the expected carbohydrate load (9). Underpinning this observation, we know that certain amino acids can be converted into glucose (gluconeogenesis), and dietary fat slows the absorption of dietary glucose causing a delayed postprandial rise. The complex biochemistry behind this and its net impact on insulin dosing is the subject of much ongoing research and debate (10).

In summary, we present here compelling evidence that a low carbohydrate diet has a positive and sustained impact on glycaemic control in a patient with type 1 diabetes. It normalised large fluctuations in daily BG readings and lead to HbA1c values in the non-diabetic range. When extrapolating the data in this report, we would stress the need for personalised nutrition recommendations. Inherent metabolic differences exist between individuals and their response to dietary modification, namely a low carbohydrate high fat/protein may be varied. Therefore, a guided approach with close dietary supervision would be recommended for future research. Further, larger scale studies are needed to establish if these findings can be extrapolated across a more diverse subsection of this population and may in principle be applicable to management of insulin-dependent patients with type 2 diabetes. Moreover, extended follow-up of this patient group should allow an accurate assessment of impact on chronic micro- and macro-vascular morbidity, as well as long-term adherence to the diet.

Declaration of interest

The authors declare that there is no conflict of interest that could be perceived as prejudicing the impartiality of the research reported.

\section{Funding}

This research did not receive any specific grant from any funding agency in the public, commercial or not-for-profit sector.

\section{Patient consent}

The patient has given written informed consented for the publication of the details contained within this document.

\footnotetext{
Patient's perspective

The switch from 150 to $200 \mathrm{~g}$ of carbohydrates a day to a low carbohydrate diet has been liberating for me. With much more stable blood sugars, I have stopped suffering from the side effects of strong fluctuations and extreme blood sugar levels: I have fewer headaches, sleep better and am thus more well rested, have more stable energy levels throughout the day and feel satiated for longer (often with less food). The dietary adjustment also allows me to exercise more spontaneously with less of an impact on
} 
my BG levels. Having fewer and smaller glycaemic excursions also means that I spend a lot less mental and emotional energy on my diabetes management and am more relaxed and less anxious and frustrated than before.

\section{Author contribution statement}

Mirjam Eiswirth: Data analysis and graph construction written contribution to body of text. Ewan Clark: Written contribution to body of text and editing. Michael Diamond: Data analysis, graph construction, written contribution to body of text and editing.

\section{References}

1 Franco OH, Steyerberg EW, Hu FB, Mackenbach J \& Nusselder W. Associations of diabetes mellitus with total life expectancy and life expectancy with and without cardiovascular disease. Archives of Internal Medicine 2007167 1145-1151. (https://doi.org/10.1001/ archinte.167.11.1145)

2 National Institute for Clinical Excellence. Type 1 Diabetes in Adults: Diagnosis and Management. NG17 ed. London: NICE, 2015.

3 Hill NE, Campbell C, Buchanan P, Knight M, Godsland IF \& Oliver NS. Biochemical, physiological and psychological changes during endurance exercise in people with type 1 diabetes. Journal of Diabetes Science and Technology 201711 529-536. (https://doi. org/10.1177/1932296816671956)
4 Jensen J, Aslesen R, Ivy JL \& Brors O. Role of glycogen concentration and epinephrine on glucose uptake in rat epitrochlearis muscle. American Journal of Physiology 1997272 E649-E655. (https;//doi. org/10.1152/ajpendo.1997.272.4.E649)

5 Krebs JD, Parry Strong A, Cresswell P, Reynolds AN, Hanna A \& Haeusler S. A randomised trial of the feasibility of a low carbohydrate diet vs standard carbohydrate counting in adults with type 1 diabetes taking body weight into account. Asia Pacific Journal of Clinical Nutrition 201625 78-84. (https://doi.org/10.6133/ apjen.2016.25.1.11)

6 Nielsen JV, Jonsson E \& Ivarsson A. A low carbohydrate diet in type 1 diabetes: clinical experience - a brief report. Upsala Journal of Medical Sciences 2005110 267-273. (https://doi.org/10.3109/2000-1967-074)

7 Thomas D \& Elliott EJ. Low glycaemic index, or low glycaemic load, diets for diabetes mellitus. Cochrane Database of Systematic Reviews 2009. (https://doi.org/10.1002/14651858.CD006296.pub2)

8 Hirsch IB \& Brownlee M. Should minimal blood glucose variability become the gold standard of glycemic control? Journal of Diabetes and its Complications 200519 178-181. (https://doi.org/10.1016/j. jdiacomp.2004.10.001)

9 Bell KJ, Smart CE, Steil GM, Brand-Miller JC, King B \& Wolpert HA. Impact of fat, protein, and glycemic index on postprandial glucose control in type 1 diabetes: implications for intensive diabetes management in the continuous glucose monitoring era. Diabetes Care 201538 1008-1015. (https://doi.org/10.2337/dc15-0100)

10 Paterson M, Bell KJ, O'Connell SM, Smart CE, Shafat A \& King B. The role of dietary protein and fat in glycaemic control in type 1 diabetes: implications for intensive diabetes management. Current Diabetes Reports 201515 61. (https://doi.org/10.1007/s11892-015-0630-5)

Received in final form 14 February 2018

Accepted 20 February 2018 